\title{
Suprapubic transvesical laparoendoscopic single-site surgery for vesicovaginal fistula repair: a case report
}

\author{
Marek Roslan, Marcin M. Markuszewski, Joanna Bagińska, Kazimierz Krajka \\ Department of Urology, Medical University of Gdansk, Poland
}

Videosurgery Miniinv 2012; 7 (4): 307-310

DOI: 10.5114/wiitm.2011.30816

\begin{abstract}
Some minimally invasive techniques have been introduced to decrease morbidity related to standard laparoscopic procedures. One such approach is laparoendoscopic single-site surgery (LESS), which can also be applied for transvesical surgery. The aim of the study was to present our initial clinical experience of using this technique for transvesical vesicovaginal fistula (VVF) repair. In August 2011, we carried out the LESS repair of a 3-mm in diameter vesicovaginal fistula on a 72-year-old woman, who failed the conservative treatment with Foley placement. The procedure was performed transvesically (percutaneous intraluminal approach) with a single-port device (4-channel, TriPort+, Olympus Winter\&IBE GMBH) via a $1.5-\mathrm{cm}$ incision made $2 \mathrm{~cm}$ above the pubic symphysis. A standard 10-mm optic and straight laparoscopic instruments were used. The fistulous tract was dissected and partially excised. The bladder and vaginal wall defects were closed in two layers with running absorbable V-Loc (Covidien, Norwalk, CT, USA) suture. Ureteral catheters were left for 5 days and the Foley catheter for 14 days. The operative time was 170 min. The blood loss was minimal. No complications were observed. The postoperative period was uneventful. During a 6-month follow-up the patient reported no involuntary discharge of urine into the vagina. Diagnostic scans revealed no presence of VVF and laboratory examination results were all within the normal range. Although substantial development of the instruments and skills is needed, the transvesical LESS vesicovaginal fistula repair appeared to be feasible and safe. Nevertheless, further experience and observations are necessary.
\end{abstract}

Key words: vesicovaginal fistula, minimally invasive surgery, laparoendoscopic single-site surgery.

\section{Introduction}

Over the past 5 years, single-port techniques, such as laparoendoscopic single-site surgery (LESS), have been applied to perform a wide range of operations, both in adults and in children, including cancer resections and live donor nephrectomies [1-3].

There are numerous applications of LESS for urological indications involving either reconstructive or ablative procedures $[4,5]$. Simple prostatectomies as well as diverticulectomies were performed transvesically via a single port placed intraperitoneally through an intra-umbilical incision $[4,6]$. A few reports describe the LESS technique performed percutaneously, directly through the bladder wall for foreign body removal, bladder cuff excision or adenomectomy [7-10]. Because of the evolution of laparoscopy over recent years, however, a number of laparoscopic approaches for treating vesicovaginal fistula (VVF) have been published $[11,12]$. Nevertheless, we have found only one report on LESS repair of VVF [13].

The aim of the study was to o present our experience of percutaneous transvesical treatment of a VVF using the TriPort+ device inserted directly into the bladder. To the best of our knowledge, this is the first 
report of a percutaneous transvesical LESS approach for VVF repair.

\section{Case report}

A 72-year-old woman presenting with continuous urinary leakage per vagina was admitted to our centre in August 2011. In May 2011, she had undergone hysterectomy because of a benign disease.

The exams demonstrated a 3-mm wide fistula between the bladder trigone and the upper part of vaginal vault (Figure 1). Microbiological examinations indicated typical chronic infection with Escherichia coli. All other laboratory parameters were within the normal range.

Because of the localization of the VVF in the vaginal apex, we initially considered the laparoscopic approach. However, as our team had carried out several LESS transvesical operations over previous years, we decided to use this technique for VVF repair with a single port introduced directly into the bladder.

The patient was placed in the lithotomy position. Subsequently, both ureters, as well as the fistulous tract, were catheterized (Figure $2 \mathrm{~A}$ ). We introduced an $18 \mathrm{~F}$ Foley catheter into the vagina and filled its balloon with water up to $30 \mathrm{ml}$. This manoeuvre

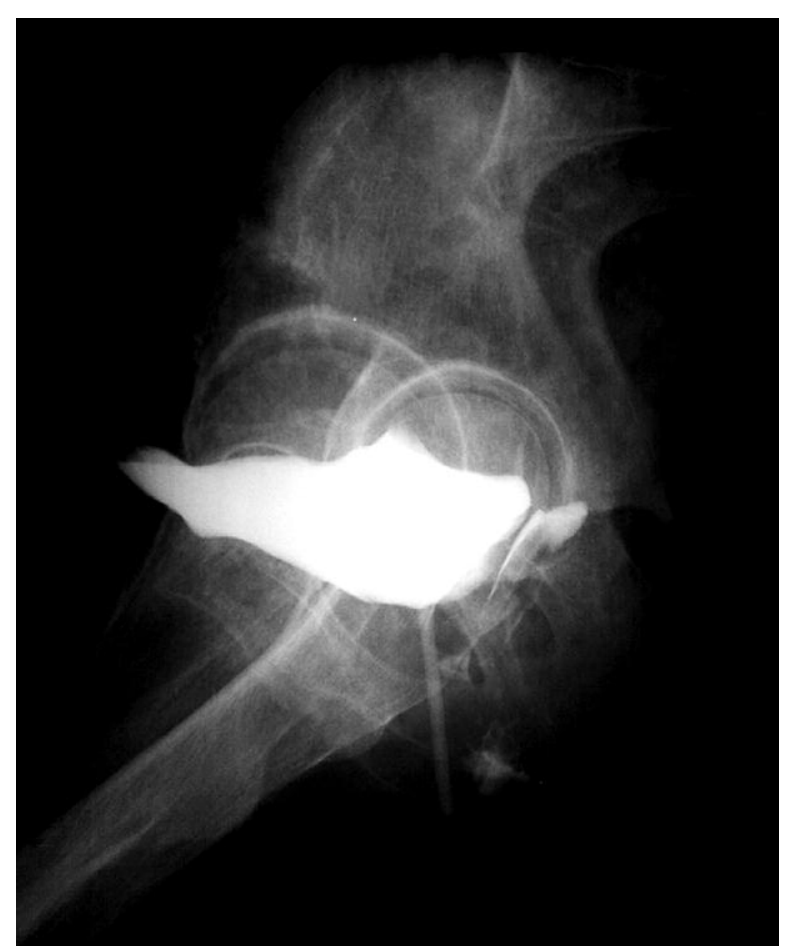

Figure 1. A cystourethrography scan shows contrast medium leaking into the vagina plugged the vagina, and decreased gas leaks during the procedure.

A $1.5-\mathrm{cm}$ longitudinal skin incision was made $2 \mathrm{~cm}$ superior to the pubic symphysis. We used two stay sutures to facilitate the insertion of the port. The introducer with the inner ring of a TriPort+ access system (Olympus Winter\&IBE GMBH, Hamburg, Germany) was inserted directly into the bladder via the skin incision under cystoscopic control. The rings of the TriPort+ were fixed to the abdominal wall area. The bladder was filled with carbon dioxide up to a pressure of $14 \mathrm{~mm} \mathrm{Hg}$.

We used a $10-\mathrm{mm}$, rigid, $30^{\circ}$ videolaparoscope (Olympus Europa GmbH, Hamburg, Germany), which was introduced through the $10-\mathrm{mm}$ channel of the TriPort+. The three 5-mm working channels were used as follows: 1) for a 5-mm standard laparoscopic dissector in the left hand, and 2) for a 5-mm monopolar rigid hook electrode or a 5-mm rigid needle-driver (Karl Storz, Tuttlingen, Germany) in the right hand. When needed, we introduced the suction tube through the third 5-mm channel of the TriPort+ or through the urethra.

The visualization inside the bladder was sufficient to recognize and control all anatomical structures. We separated the fistula from the bladder mucosa by a circular cut using a hook electrode (Figures 2 B, C). The superficial rim of the fistulous tract was resected, and either the vaginal wall or the bladder defect was closed tightly with an absorbable 3/0 running suture (The V-Loc ${ }^{\text {TM }} 90$ Absorbable Wound Closure Device, Covidien, Norwalk, CT, USA) (Figures 2 D, E). The integrity of the bladder was confirmed by filling it with $200 \mathrm{ml}$ of saline. Betadine-soaked roller gauze was inserted into the vagina for one day. The skin incision was sutured with two stitches. The procedure was completed successfully with no extra port insertion. The operative time was $170 \mathrm{~min}$, and the blood loss was insignificant. The postoperative period was uneventful. On the fifth postoperative day, ureteric catheters were removed and the patient was discharged home. She presented no vaginal leakage on discharge. An 18 F Foley catheter was retained for 2 weeks. Quinolone oral intake was administered for 3 weeks and pelvic rest was recommended for 2 months.

Five weeks after surgery, diagnostic scans (urethrocystography) revealed no presence of VVF (Figure 3). During a 4-month follow-up, the patient remained continent, and laboratory examination results were all within the normal range. 

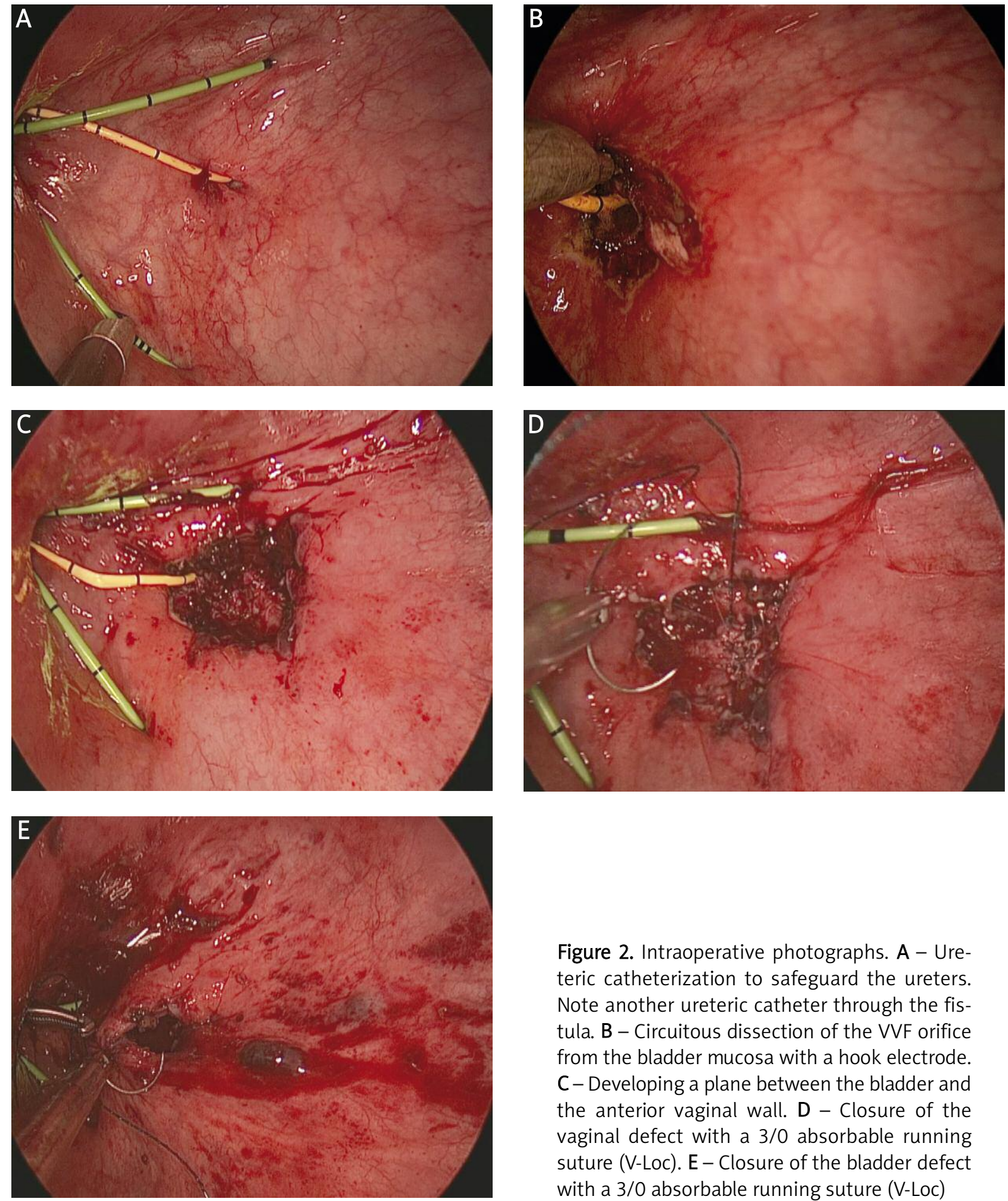

Figure 2. Intraoperative photographs. A - Ureteric catheterization to safeguard the ureters. Note another ureteric catheter through the fistula. B - Circuitous dissection of the VVF orifice from the bladder mucosa with a hook electrode. $\mathrm{C}$ - Developing a plane between the bladder and the anterior vaginal wall. D - Closure of the vaginal defect with a 3/0 absorbable running suture (V-Loc). E - Closure of the bladder defect with a 3/0 absorbable running suture (V-Loc)

\section{Discussion}

To date, there is no consensus on what approach is the best for VVF repair. At present, laparoscopy and single-port procedures tend to replace open surgery,

with comparable results [11-14]. These procedures mostly follow the O'Conor technique [15]. However, simpler techniques are also available [16].

We also considered the extravesical approach for LESS repair of VVF, according to Abdel-Karim et al. 


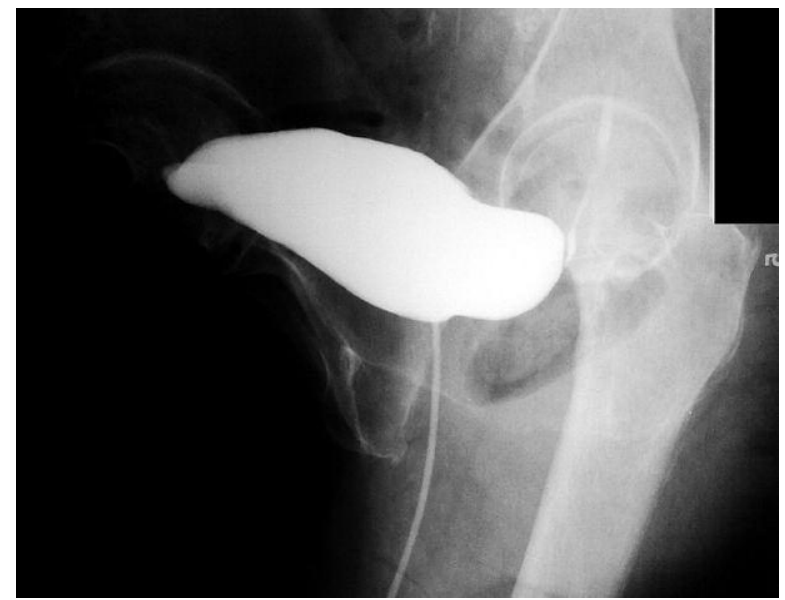

Figure 3. A postoperative cystogram obtained 7 weeks later does not show any leak

[13], but the abdominal access often requires extensive and time-consuming adhesiolysis, and, because we were familiar with different transvesical LESS procedures, we decided to use this approach in our patient.

After adequate dissection of the bladder from the vaginal wall, both openings of the fistula were closed with separate running V-Loc sutures, and a watertight closure was achieved. The advantages of our method are (i) the reduction of the number of ports, (ii) avoiding bivalving the bladder, and (iii) the possibility to use the transurethral access to facilitate intravesical dissection or suturing. The use of a barbed suture (V-Loc) also eliminates the need for tying knots and the number of instruments used. This also reduces the operative time.

The disadvantages are similar to those in other LESS procedures. This approach is probably not applicable for larger and complicated fistulae.

The authors realize that one case is insufficient to define more general conclusions. Nevertheless, based on our previous experience in the field on laparoscopy and transvesical laparoendoscopy, we find this approach to be a logical, minimally invasive, and simple access for either ablative or reconstructive procedures in the bladder.

Our positive experience in this approach shows that percutaneous transvesical LESS vesicovaginal fistula repair is a feasible, effective and safe procedure, but a larger number of patients is needed to thoroughly evaluate this treatment.

\section{References}

1. Urbanowicz W, Sulislawski J, Wolnicki M. Single incision laparoscopic surgery in children - a preliminary report. CEJ Urol 2010; 63: 132-4.

2. Michalik M, Orłowski M, Frask A, et al. LESS (laparo-endoscopic single-site surgery) right hemicolectomy. Videosurgery Miniinv 2009; 4: 164-7.

3. Ganpule A, Dhawan D, Kurien A, et al. Laparoendoscopic singlesite donor nephrectomy: a single-center experience. Urology 2009; 74: 1238-40.

4. Desai MM, Berger AK, Brandina R, et al. Laparoendoscopic single-site surgery: initial hundred patients. Urology 2009; 74: 805-12.

5. Gettman M, White MW, Aron M, et al. Where do we really stand with LESS and NOTES? Eur Urol 2011; 59: 231-4.

6. Stolzenburg JU, Do M, Kallidonis P, et al. Laparoendoscopic single-site bladder diverticulectomy: technique and initial experience. J Endourol 2011; 25: 85-90.

7. Ingber M, Stein R, Rackley R, et al. Single-port transvesical excision of foreign body in the bladder. Urology 2009; 74: 1347-50.

8. Roslan M, Markuszewski M, Gibas A, et al. Laparoendoscopic single-site transvesical removal of midurethral polypropylene sling eroded into the bladder. Videosurgery Miniinv 2011; 6: 111-4.

9. Sotelo R, Ramirez D, Carmona O, et al. A novel technique for distal ureterectomy and bladder cuff excision. Acta Urol Esp 2011; 35: 168-74

10. Desai MM, Fareed K, Berger AK, et al. Single-port transvesical enucleation of the prostate: a clinical report of 34 cases. BJU Int 2010; 105: 1296-300.

11. Shah SJ. Laparoscopic transabdominal transvesical vesicovaginal fistula repair. J Endourol 2009; 23: 1135-7.

12. Guzen AS, Teber D, Canda AE, Rassweiller J. Transperitoneal laparoscopic repair of iatrogenic vesicovaginal fistulas: Heilbronn experience and review of the literature. J Endourol 2009; 23: 475-9.

13. Abdel-Karim AM, Moussa A, Elsalmy S. Laparoendoscopic singlesite surgery extravesical repair of vesicovaginal fistula: early experience. Urology 2011; 78: 567-71.

14. Sotelo R, Mariano MB, García-Segui A, et al. Laparoscopic repair of vesicovaginal fistula. J Urol 2005; 173: 1615-8.

15. O'Conor VJ, Sokol JK, Bulkley GJ, et al. Suprapubic closure of vesicovaginal fistula. J Urol 1973; 109: 51-4.

16. Elkins T, Thompson J. Lower urinary tract fistulas. In: Walters $M$, Karram M (eds.). Urogynecology and Reconstructive Pelvic Surgery. St Louis, Mo: Mosby 1999; 355-66.

Received: 12.03.2012, Revised: 5.04.2012, Accepted: 9.05.2012. 